The University of Southern Mississippi

The Aquila Digital Community

Faculty Publications

$5-1-2004$

\title{
Diode Laser Microwave Induced Plasma Cavity Ringdown Spectrometer: Performance and Perspective
}

\author{
Chuji Wang \\ Mississippi State University, wang@dial.msstate.edu \\ Sudip P. Koirala \\ Mississippi State University \\ Susan T. Scherrer \\ Mississippi State University \\ Yixiang Duan \\ Los Alamos National Laboratory \\ Christopher B. Winstead \\ University of Southern Mississippi
}

Follow this and additional works at: https://aquila.usm.edu/fac_pubs

Part of the Physics Commons

\section{Recommended Citation}

Wang, C., Koirala, S. P., Scherrer, S. T., Duan, Y., Winstead, C. B. (2004). Diode Laser Microwave Induced Plasma Cavity Ringdown Spectrometer: Performance and Perspective. Review of Scientific Instruments, 75(5), 1305-1313.

Available at: https://aquila.usm.edu/fac_pubs/3283

This Article is brought to you for free and open access by The Aquila Digital Community. It has been accepted for inclusion in Faculty Publications by an authorized administrator of The Aquila Digital Community. For more information, please contact Joshua.Cromwell@usm.edu. 


\title{
Diode laser microwave induced plasma cavity ringdown spectrometer: Performance and perspective
}

\author{
Chuji Wang, ${ }^{\text {a) }}$ Sudip P. Koirala, and Susan T. Scherrer \\ Diagnostic Instrumentation and Analysis Laboratory, Mississippi State University, 205 Research Boulevard, \\ Starkville, Mississippi 39759 \\ Yixiang Duan \\ Los Alamos National Laboratory, C-ACS, MS K-484, Los Alamos, New Mexico 87545 \\ Christopher B. Winstead \\ Department of Physics and Astronomy, University of Southern Mississippi, Box 5046, Hattiesburg, \\ Mississippi 39406
}

(Received 28 October 2003; accepted 6 February 2004; published 26 April 2004)

\begin{abstract}
Recent studies combining an atmospheric-pressure plasma source (inductively coupled plasma or microwave induced plasma) with cavity ringdown spectroscopy (plasma-CRDS) have indicated significant promise for ultra-sensitive elemental measurements. Initial plasma-CRDS efforts employed an inductively coupled plasma as the atomization source and a pulsed laser system as the light source. In an effort to improve the portability and reduce the cost of the system for application purposes, we have modified our approach to include a compact microwave induced plasma and a continuous wave diode laser. A technique for controlling the coupling of the continuous wave laser to the ringdown cavity has been implemented using a standard power combiner. No acouto-optic modulator or cavity modulation is required. To test the system performance, diluted standard solutions of strontium $(\mathrm{Sr})$ were introduced into the plasma by an in-house fabricated sampling device combined with an ultrasonic nebulizer. $\mathrm{SrOH}$ radicals were generated in the plasma and detected using both a pulsed laser system and a diode laser via a narrow band transition near 680 $\mathrm{nm}$. The experimental results obtained using both light sources are compared and used for system characterization. The ringdown baseline noise and the detection limit for $\mathrm{Sr}$ are determined for the current experimental configuration. The results indicate that a plasma-CRDS instrument constructed using diode lasers and a compact microwave induced plasma can serve as a small, portable, and sensitive analytical tool. (C) 2004 American Institute of Physics. [DOI: 10.1063/1.1711150]
\end{abstract}

\section{INTRODUCTION}

Following its introduction, ${ }^{1}$ cavity ringdown spectroscopy (CRDS) was rapidly implemented for the study of numerous species in a variety of environments. ${ }^{2-7}$ Research and applications relevant to CRDS have expanded from the initial concept to include other light sources, ${ }^{8-11}$ optical cavities, ${ }^{12-16}$ methods of cavity excitation and shutoff, ${ }^{17-23}$ and combinations with other traditional instruments. ${ }^{24-29} \mathrm{Al}-$ though the development of ringdown-based commercial instrumentation is still in its early stages, this technology has already shown much promise in many application areas such as semiconductor manufacturing, ${ }^{30}$ environmental monitoring, ${ }^{31,32}$ and medical diagnostics. ${ }^{33,34}$ One such application is analytical atomic spectrometry using CRDS combined with an atmospheric-pressure inductively coupled plasma (ICP). The ICP-CRDS technique has demonstrated significant potential as an analytical instrument for ultrasensitive elemental and isotopic measurements. ${ }^{25-27}$ Detection limits for several elements and isotopes measured using the ICP-CRDS technique range from the parts-per-billion to

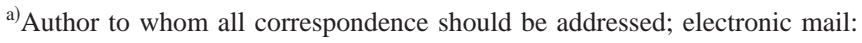
wang@dial.msstate.edu
}

hundreds of parts-per-trillion levels. These results, obtained using a pulsed dye laser, were directly compared with those obtained by ICP-laser induced fluorescence (ICP-LIF), ${ }^{35}$ ICP-mass spectrometry, ${ }^{36}$ and ICP-optical emission spectrometry. ${ }^{36}$ More recently, an atmospheric-pressure compact microwave induced plasma (MIP) has been introduced as the atomization/ionization source coupled with CRDS (MIP-CRDS) ${ }^{29}$ For applications requiring portability, the replacement of the pulsed laser system with a compact light source is an essential next step.

This article describes the use of a continuous wave $(\mathrm{CW})$ diode laser to reduce the size and cost of plasma-CRDS and to produce a compact instrument configuration-namely, diode laser-MIP-CRDS. It should be noted that without a specification of ICP-CRDS or MIP-CRDS, the plasmaCRDS includes the both in the following text. The ringdown cavity excitation is controlled using a configuration based on a standard power combiner. To test the system performance, diluted standard solutions of strontium were introduced into the plasma by an in-house fabricated sampling device combined with an ultrasonic nebulizer. $\mathrm{SrOH}$ radicals were generated in the plasma and detected via a narrow band transition near $680 \mathrm{~nm}$. Extensive ringdown measurements of $\mathrm{SrOH}$ have been performed to test the instrument. Estimated 
detection limits for lead, strontium, and uranium are also presented.

\section{PLASMA-CRDS PRINCIPLES FOR ELEMENTAL MEASUREMENTS}

In an earlier report, the use of CRDS in combination with an inductively coupled plasma was described in detail. ${ }^{26}$ The use of a MIP as an atomization source and the substitution of a diode laser in place of a pulsed dye laser system substantially modify the experimental configuration and the operational characteristics of the system. However, basic mathematical and experimental considerations remain similar and will be only briefly summarized here. The ringdown time for the plasma-CRDS experiments can be expressed as

$$
\tau=\frac{d}{c\left[1-R+\beta_{\text {plasma }} l+\beta_{\text {air }}(d-l)+\text { absorbance }\right]},
$$

where $d$ is the cavity mirror separation, $R$ is the mirror reflectivity, $\beta_{\text {plasma }}$ and $\beta_{\text {air }}$ are broadband scattering coefficients in the plasma and open air cavity, $c$ is the speed of light, $l$ is the optical path-length through the plasma, and the absorbance is due to absorption by analyte species in the plasma. Although the use of a continuous wave light source for $\mathrm{CRDS}^{37-39}$ alters the scheme for coupling light into the cavity (as described below), the ringdown time equation above remains valid. The above equation is not ordinarily used in data analysis, but is provided to highlight the effect of various loss mechanisms (evident in the denominator) on the ringdown time. In practice, because the scattering coefficients vary slowly as a function of wavelength, such losses are simply incorporated into an effective reflectivity value $R_{\text {eff }}$, and the ringdown time equation can be expressed as

$$
\tau=\frac{d}{c\left(1-R_{\mathrm{eff}}+\text { absorbance }\right)} .
$$

At a given wavelength, $R_{\text {eff }}$ is easily determined by measuring the ringdown time $\tau_{0}$ with no analyte presented in the plasma (i.e., absorbance is zero).

Because the density of absorbers varies as a function of position in the plasma and the absorption cross section of a given optical transition is a function of frequency, an appropriate expression for the absorbance is

$$
\text { absorbance }=2 \int_{-\infty}^{\infty} \int_{0}^{l / 2} \sigma(\nu) n(r) d r d \nu,
$$

where $r$ represents the position along the laser beam path through the plasma of diameter $l, n(r)$ is the concentration of absorbers (assumed to be radially symmetric), $v$ is the optical frequency of the laser, and $\sigma(\nu)$ is the absorption crosssection for an absorption line. Due to strong homogeneous and inhomogeneous broadening, the lineshape of the absorption line is a Voigt profile, and the absorption cross section can be represented as ${ }^{40,41}$

$$
\sigma_{i j}(\nu)=\frac{g_{j}}{g_{i}} \frac{\lambda^{4}}{4 \pi^{2} c} \frac{A_{j i}}{\Delta \lambda_{D}} V(a, 0)(\pi \ln 2)^{1 / 2},
$$

where, $g_{j}$ and $g_{i}$ are the upper and lower state degeneracies, $\lambda$ is the transition wavelength, $c$ is the speed of light, and $A_{j i}$ is the spontaneous emission transition rate. For most atomic/ ionic transitions, these parameters are well documented in the literature. $^{42} \Delta \lambda_{D}$ is the Gaussian component of the broadened linewidth due to Doppler broadening and is determined by $\Delta \lambda_{D}=7.16 \times 10^{-7} \lambda_{0} \sqrt{(T / M)}$, where $\lambda_{0}$ is the central wavelength of the line, $T$ the absolute temperature $(\mathrm{K})$, and $M$ the atomic mass (a.u.). ${ }^{43} V(a, 0)$ is the Voigt function determined by and dependent on the $a$ parameter

$$
\begin{aligned}
& V(a, 0)=\frac{a}{\pi} \int_{-\infty}^{\infty} \frac{e^{-t^{2}}}{a^{2}+t^{2}} d t, \\
& a=\sqrt{\ln 2} \frac{\Delta \lambda_{L}}{\Delta \lambda_{D}} .
\end{aligned}
$$

$\Delta \lambda_{L}$ is the Lorentzian component of the broadened linewidth due to homogeneous mechanisms such as collisional broadening. When Doppler broadening dominates, e.g., when $a$ $=0.1$, the value of $V(a, 0)$ is close to 1 ; for a Voigt lineshape, e.g., $a=1.2, V(a, 0)=0.38$.

Due to the heterogeneity of plasma parameters such as temperature and electron density, the lineshape is a function of position in the plasma. Strictly, the absorption cross section is a thus function of position as well as optical frequency, implying that spatially resolved lineshape data is needed to most accurately calculate the cross section. In practice, the measured lineshape is typically the spatially averaged, integrated lineshape along the particular line-ofsight; therefore, an averaged value of the cross section is obtained. Using this line-of-sight averaged value for the cross section, then Eqs. (2) and (3) can be simplified and combined to obtain

$$
\sigma(\nu) n(x) l=\frac{d}{c}\left(\frac{1}{\tau}-\frac{1}{\tau_{0}}\right),
$$

where $\tau_{0}$ incorporates all background losses observed when no analyte is present. Using the experimental lineshape data to determine the average cross-section allows for the measurement of the average density of atoms or ions in the lower state of the transition being probed [i.e., $n(x)$ where $x$ is the lateral position at which the beam crosses the plasma]. The radial density, $n(r)$ in Eq. (3), can be obtained through Abel inversion $^{26,44-47}$ for the conversion of the lateral measurements. Such measurements are quite useful for diagnostic purposes and for estimating system sensitivity (as described below). It is important to be aware of the drawbacks of using the line-of-sight measurements. For the purpose of plasma diagnostics, such as measuring the distribution maps of electron density, gas kinetic temperature, and analyte/species density, the line-of-sight measurement may generate a large deviation. $^{26,48,49}$ However, for the analytical application in which the detection sensitivity is normally concerned, the line-of-sight measurement is still applicable and quite practical. $^{26,27,48,50}$ 


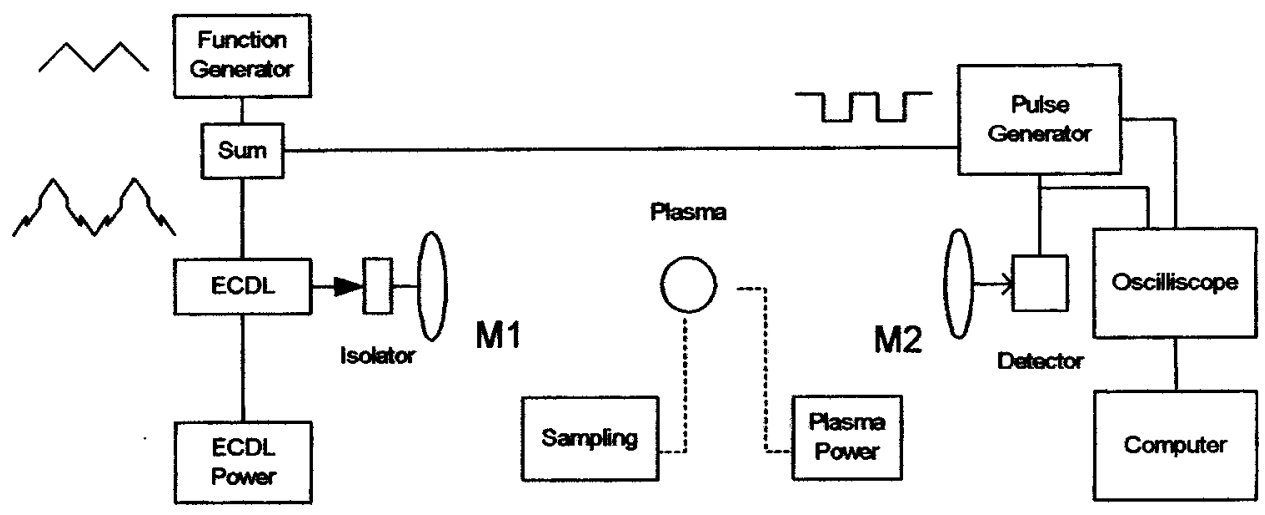

FIG. 1. A schematic of the diode laser-microwave induced plasmacavity ringdown spectrometer.

\section{EXPERIMENTAL DETAILS}

Figure 1 presents a schematic for the plasma-CRDS instrument implemented with an external cavity diode laser. The system consists of six major components: the light source, plasma source, sampling device, optical cavity, data acquisition, and electronic control. Each component is described in the following.

\section{A. Light source}

Continuous wave radiation was provided by an external cavity diode laser (ECDL) (New Focus, Vortex TLB 6009). The central wavelength of the ECDL output is $682.88 \mathrm{~nm}$ with a $70 \mathrm{GHz}$ fine-tuning range without mode hop. Fine frequency modulation can be achieved by modulating either the laser diode current or the voltage applied to a piezoelectric actuator mounted on the ECDL mirror. The laser linewidth is less than $100 \mathrm{MHz}\left(\sim 0.003 \mathrm{~cm}^{-1}\right)$ and the maximum laser power is $10 \mathrm{~mW}$. The single mode laser beam is passed through a $45 \mathrm{~dB}$ optical isolator (OFR IO-3-682-LP) and coupled into a single mode optical fiber. The output of the fiber is collimated and injected into the ringdown cavity. A pulsed laser system was also used to compare light source effects and the system performance with different laser sources. The pulsed radiation was generated by a narrow linewidth dye laser (Radiant NarrowScan G-R) pumped at $532 \mathrm{~nm}$ using a $20 \mathrm{~Hz}$ repetition rate Nd:YAG laser (Continuum Powerlite 8020). The pulse duration is 5-7 ns. In the single grating configuration used for this work, the dye laser linewidth at $680 \mathrm{~nm}$ is $\sim 0.08 \mathrm{~cm}^{-1}$ as measured by a 0.25 $\mathrm{cm}^{-1}$ Fabry-Pérot etalon with a central wavelength of 700 $\mathrm{nm}$. The minimum scanning step of the dye laser is 0.0006

TABLE I. MIP operation conditions.

\section{Plasma}

Microwave power

Plasma supporting gas flow rate

Plasma central gas flow rate

Sampling

Sample up-take rate

Heating temperature of the ultrasonic nebulizer chamber

Cooling temperature of the ultrasonic nebulizer desolvator

Heating temperature of the membrane device

$\mathrm{N}_{2}$ gas flow rate in the drier $\mathrm{nm}$. The laser beam is approximately mode-matched into the ringdown cavity using a telescope/spatial filter system consisting of two focusing lenses and a pinhole. For the cavity mirrors employed, a few $\mu \mathrm{J}$ of laser pulse energy is sufficient to perform the reported measurements.

\section{B. Plasma source}

The atmospheric-pressure toroidally shaped microwave induced plasma can be operated with a power ranging from tens of watts to several hundred watts. The plasma torch is constructed from three coaxial brass tubes and is connected to a $2450 \mathrm{MHz}$ microwave power supply through a $1 \mathrm{~m}$ coaxial cable. Typical operational parameters for the MIP are summarized in Table I. Figure 2 presents an image of the plasma obtained using a digital camera. The plasma consists of three distinctive regions: a narrow portion at the top, a thin waist, and a triangular bottom. The best location for ringdown measurements in terms of detection sensitivity is located in the upper portion of the triangular region. Advantages of using a MIP instead of an ICP have been discussed extensively in our previous work. ${ }^{28,29}$ Briefly, the MIP requires less electrical power and operating gas and also does not require a cooling system, important advantages when fieldability and portability are needed. We have also found that the MIP source is more stable than the ICP source for CRDS measurements. Efforts to extend the interaction pathlength of the laser beam and sample in the MIP are also underway.

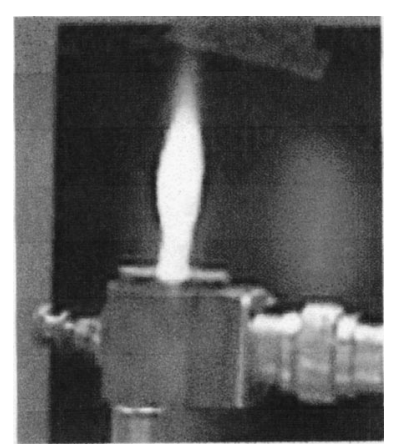

$0.35 \mathrm{~L} / \mathrm{min}$

$0.50 \mathrm{~L} / \mathrm{min}$

$1.0 \mathrm{ml} / \mathrm{min}$

$140{ }^{\circ} \mathrm{C}$

$-5^{\circ} \mathrm{C}$

$80{ }^{\circ} \mathrm{C}$

$0.5 \mathrm{~L} / \mathrm{min}$

FIG. 2. A typical picture of the microwave induced plasma operating with argon carrier gas. 


\section{Sampling device}

Samples containing various concentrations of strontium were prepared by diluting a standard solution with a $2 \%$ nitric acid solution. The working solution is pumped into an ultrasonic nebulizer (CETAC U-5000AT ${ }^{+}$) to generate an aerosol, which is subsequently carried by argon gas through a drying device constructed using a membrane desolvator. The dried gas stream was then injected into the plasma torch. The overall sample conversion efficiency from liquid to gas is about $10 \%$.

\section{Ringdown cavity}

A $780 \mathrm{~mm}$ long cavity is formed by two plano-concave mirrors with a $1 \mathrm{~m}$ radius of curvature (Newport Corporation, Irvine, CA). The center wavelength for the mirrors is specified to be $620 \mathrm{~nm}$ with a reflectivity of $99.99 \%$. For the $680 \mathrm{~nm}$ wavelength used in this effort, the maximum reflectivity of the mirrors is $99.9 \%$ and the effective reflectivity ${ }^{26,29}$ is $99.85 \%$ when the plasma is on. The spacing between the longitudinal modes of this cavity is 0.0064 $\mathrm{cm}^{-1}$, or $192.3 \mathrm{MHz}$. The location of the plasma torch between the ringdown cavity mirrors can be continuously adjusted in both the vertical and lateral directions using a laboratory fabricated mount with an estimated position accuracy of $\pm 0.5 \mathrm{~mm}$.

\section{E. Data acquisition system}

A photomultiplier tube (PMT-Hamamatsu R928) connected to a digital oscilloscope (Tektronix, TDS 460A) is used to digitize the ringdown waveform. The oscilloscope is interfaced to a computer via a general purpose interface bus (IEEE 488) interface. Custom laboratory-developed software is used for real-time determination of the ringdown time constant. A $10 \mathrm{~nm}$ band-pass interference filter was mounted in the front of the PMT to reject optical emissions from the plasma while transmitting the laser wavelength. In addition to optimizing the cavity alignment by maximizing the ringdown time constant, the alignment of the ringdown cavity and single exponential behavior of the ringdown decay are quantified by fitting different segments of the ringdown waveform over times ranging from one to three ringdown times. When the ringdown times obtained by fitting different segments of the decay waveform differ by $\sim 1 \%$ or less, the system is considered to be aligned and the use of single exponential fits is considered to be justified. In data collection, each data point typically consists of an average of 50100 ringdown events.

\section{F. Electronic control}

For pulsed laser data acquisition, the bandwidth of the laser is several times larger than the longitudinal mode spacing of the ringdown cavity. Light is always coupled into the cavity on each laser pulse so that no extreme care is required to achieve cavity excitation. However, the bandwidth of the diode laser used here is much smaller than the cavity mode spacing, implying that the laser must be tuned into resonance with a cavity mode in order to couple light into the cavity. Ringdown cavity energy excitation is achieved by laser frequency modulation through applying a sawtooth waveform

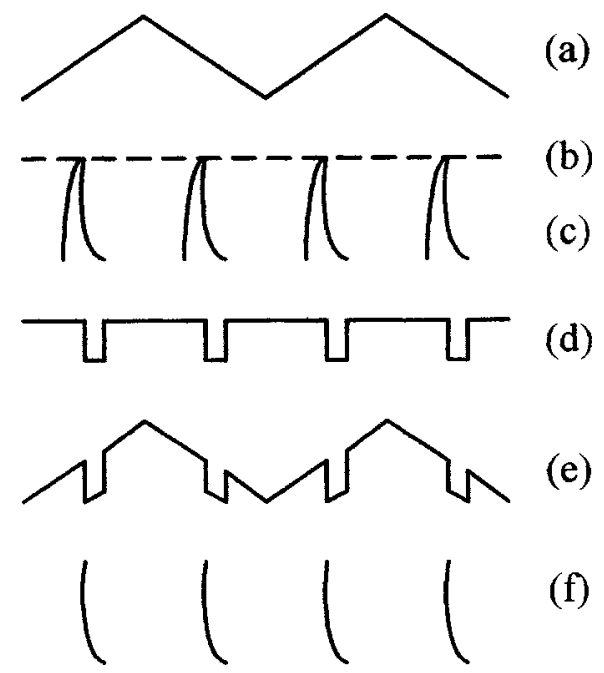

FIG. 3. A schematic of the method of controlling the coupling of the continuous wave laser to the ringdown cavity. (a) Sawtooth signal from the function generator; (b) trigger threshold; (c) energy buildup inside the cavity; (d) negative pulses from the pulse generator; (e) resultant modulation signal; and (f) ringing down of the signal.

$(< \pm 4.5 \mathrm{~V})$, generated by a function generator (HP 33120A) to the piezoelectric actuator of the laser control. The modulation rate was optimized at $10 \mathrm{~Hz}$ in order to obtain the best ringdown baseline stability. The waveform is applied through a power combiner as described below. The sawtooth waveform modulation has the effect of sweeping the laser frequency at a $10 \mathrm{~Hz}$ rate. As the laser frequency comes into resonance with a cavity mode, light couples into the cavity resulting in a buildup of the light intensity circulating in the cavity. A small fraction of this light is transmitted through the cavity mirror and is detected by the PMT. In addition to being connected to the input of the digital oscilloscope, the PMT output is connected to the high impedance trigger input of a pulse generator (Stanford Research Systems DG 535). Once the cavity is excited and the signal intensity from the PMT exceeds a trigger threshold set in the pulse generator, a negative square wave signal is generated by the pulse generator and summed in a power combiner (Mini-circuits power combiner ZFRSC-2050) with the laser frequency sweep control signal. The addition of the negative square wave to the sawtooth waveform disturbs the pattern of the frequency modulation, shifting the laser frequency out of resonance with the cavity mode and shutting off the cavity excitation. The diagram of the controlling procedure of this method outlined above is depicted in Fig. 3. The negative square wave voltage only needs to be large enough to shift the laser frequency out of resonance, an adjustment made easily by changing the voltage while monitoring the ringdown signal. Several methods have been employed to shutoff cavity excitation in continuous wave CRDS. However, for absorption lines significantly broader than the cavity mode spacing, this particular route provides a very simple and cost-effective method that has proven to be extremely practical and easy to implement with both external cavity and distributed feedback diode lasers. ${ }^{22,23}$ When the absorption linewidth is considerably broader than the cavity mode spacing (as is the case here and in many atmospheric pressure 
applications), a cavity mode near the absorption maximum can be excited without the need for tuning the cavity by modulating the cavity length. A more detailed description of this method is in preparation for future publication. ${ }^{23}$

\section{RESULTS AND DISCUSSION}

\section{A. Diode laser MIP-CRDS baseline noise}

The ringdown baseline noise is defined as $\sigma_{\tau} / \bar{\tau}$, where $\sigma_{\tau}$ is the standard deviation of the ringdown time and $\bar{\tau}$ is the averaged ringdown time. In plasma-CRDS, additional ringdown baseline noise is caused by the plasma source. The baseline noise varies with different plasma sources (e.g., an ICP or an MIP) or even with the same plasma source when operating under different conditions (e.g., plasma power, gas flow rate, carrying gas, and the location of the probed volume in the plasma). In ICP-CRDS, the relatively larger gas flow rates and higher plasma power cause the baseline noise to be considerably higher when the plasma is on. Typically, the baseline noise increases by a factor of 3-4 times when the plasma is on. However, in MIP-CRDS, the lower plasma power and small gas flow rates allow more stable ringdown baselines. In fact, the presence or absence of the plasma does not affect the baseline noise when the system is optimized. The baseline ringdown time may decrease when the plasma is operating, but the overall baseline stability is not affected.

The advantage of the improved baseline noise can be observed by rearranging Eq. (8) in Sec. I as follows:

$$
\text { Absorbance }=\sigma n l=(1-R) \frac{\Delta \tau}{\tau},
$$

where, $\sigma, n, l$, and $R$ are as defined in Sec. II; $\tau$ is the ringdown time with an analyte present, and $\Delta \tau$ is the difference between $\tau_{0}$ (without analytes) and $\tau$. Thus, for a given mirror reflectivity, the minimum detectable absorbance of the system depends upon the minimum detectable change in ringdown time $\Delta \tau / \tau$. In accordance with standard practice for analytical applications, we define the minimum detectable change to be three standard deviations of the baseline so that the minimum detectable absorbance is $(1-R) 3 \sigma_{\tau} / \tau$. A typical baseline noise obtained from the MIP-CRDS with the external cavity diode laser is $0.2 \%$, which is generated by averaging 100 ringdown events for each data point; the average $\tau$ is $8.5 \mu$ s with a standard deviation of $0.017 \mu$ s. With such a baseline noise and the effective mirror reflectivity of $99.85 \%$ used in this work, the minimum detectable absorbance is $9 \times 10^{-6}$.

\section{B. Ringdown measurements on standard Sr solution sample}

For absorption based spectrometric methods, most elemental spectral transitions of interest are found at ultraviolet wavelengths. However, at present diode lasers are readily available only at red to infrared wavelengths and over a small spectral range near $400 \mathrm{~nm}$. Technologies available include both temperature controlled diode lasers (red to nearIR) and external cavity diode lasers (blue and red to nearIR). In spite of their limited wavelength coverage, today's

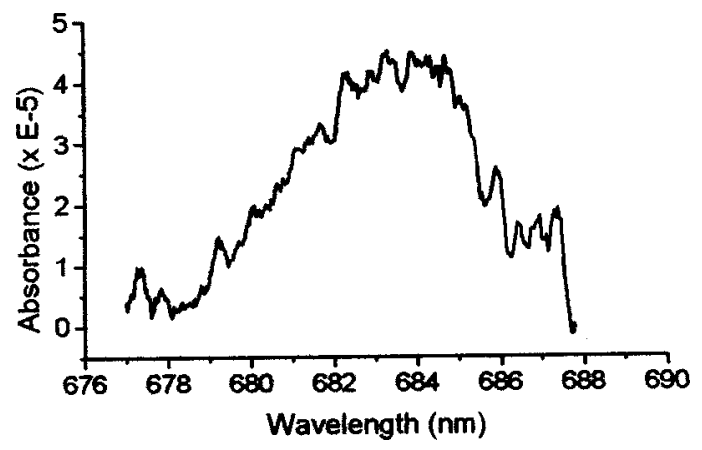

FIG. 4. Ringdown spectrum of $\mathrm{SrOH}$ obtained by the pulsed laser-MIPCRDS. Sr concentration: $25 \mathrm{ppm}$. Light source: pulsed laser system with linewidth $\sim 0.08 \mathrm{~cm}^{-1}$ in the wavelength region used in this work.

diode lasers do allow some significant problems to be addressed. For example, important isotopic measurements for actinide species such as uranium and plutonium can be performed with readily available diode laser sources. As part of such an effort, a single wavelength ECDL operating at 682 nm was procured.

For system characterization and basic operational optimization, it is usually wise to use a nonradioactive test species prior to the introduction of radioactive elements. Here, strontium standard solutions were selected for this purpose. Near $682 \mathrm{~nm}$, strontium has three atomic (Sr I) transition lines at 679.1, 687.8, and $689.3 \mathrm{~nm}$. However, once $\mathrm{Sr}$ is introduced into the plasma, the $\mathrm{SrOH}$ radical is readily generated and is observable via a narrow absorption band (also near $682 \mathrm{~nm}$ ) attributed to the $\tilde{A}-\tilde{X}$ system, which was extensively studied using laser induced fluorescence (LIF) dispersed spectra under low pressure conditions and the rotational analysis was performed. ${ }^{51}$ Several electronic transition systems of $\mathrm{SrOH}$ were also observed by LIF under supersonic jet conditions where $\mathrm{SrOH}$ was formed by laser ablation of Sr metal..$^{52}$ Under atmospheric-pressure plasma conditions, the plasma induced line broadening (pressure and temperature broadening) causes the $\mathrm{SrOH}$ band to appear diffuse. Absorption due to any remaining $\mathrm{Sr}$ is not observable as the three atomic transitions are hidden by the diffuse band (see Fig. 4). The spectrum in Fig. 5 looks "noisy," but the features superimposed on the contour are repeatable. Furthermore, the contour of the spectrum in Fig. 4 is similar to the one observed in flames. ${ }^{53}$ No detailed analysis of the spec-

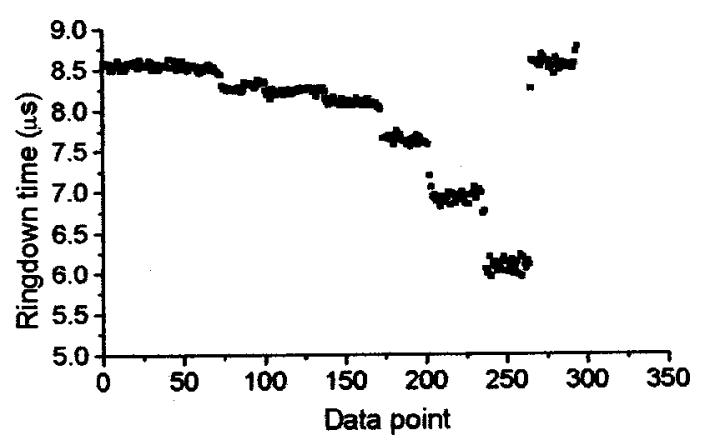

FIG. 5. Ringdown time vs $\mathrm{Sr}$ concentration. The concentration range tested: 0.5-20 ppm. Experimental configuration: external cavity diode laser with MIP. Each ramp has a single mode buildup. Light source: New focus external cavity diode laser Vortex TLB 6009. 


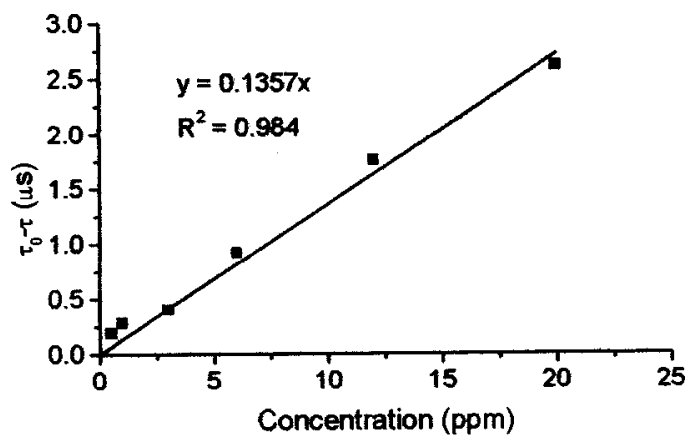

FIG. 6. A typical calibration curve. The detection limit is $375 \mathrm{ppb}$ based on the $3 \sigma$ criteria; $\sigma_{\tau}=0.017 \mu \mathrm{s}$.

trum was carried out in this work. Although ringdown spectra of $\mathrm{SrOH}$ do not directly measure the absorption of the $\mathrm{Sr}$ element, the measurements described below indicate that $\mathrm{SrOH}$ serves as an excellent species for analytical determination of the Sr concentration of the laboratory solutions.

Figure 5 presents the ringdown time versus sample solution concentration obtained using the diode laser MIP-CRDS system. The working solutions with different concentrations are prepared by diluting a standard $\mathrm{Sr}$ solution of $1000 \mathrm{ppm}$ with $2 \%$ nitric acid solution. The laser crosses the plasma at a height $13 \mathrm{~mm}$ above the torch surface and passes through the center of the plasma. Each data point represents an average of 50 ringdown events. The detection limits for $\mathrm{Sr}$ can be determined from the ringdown measurements of $\mathrm{SrOH}$ presented in Fig. 5. The calibration curve is plotted in Fig. 6. The fitted line was forced to pass the zero point. The detection limit is determined by $3 \sigma_{\tau} / k$, where $\sigma_{\tau}$ is the standard deviation of the ringdown time and $k$ is the slope of the fitted line in Fig. 6. Based on the standard $3 \sigma$ criteria described above, the derived detection limit is $375 \mathrm{ng} / \mathrm{ml}$. This detection limit, though not extensively optimized for the effects of varying the plasma parameters and certainly not indicative of the ultimate performance of the system, nevertheless provides a good figure of merit for comparison with pulsed laser experiments. Figure 7 depicts the $\mathrm{SrOH}$ absorbance versus measurement height in the plasma. It can be seen from Fig. 7 that the measured absorbance is sensitive to the plasma height and that the strongest absorption is obtained at $15 \mathrm{~mm}$ above the torch. Figure 8 presents the absorbance as a function of the plasma lateral position as the laser passes through

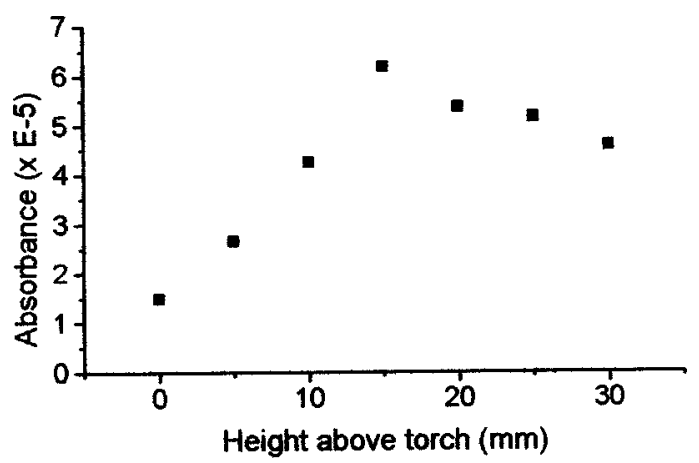

FIG. 7. Ringdown signal intensity vs plasma height. Sr concentration: 25 ppm. Microwave power: $120 \mathrm{~W}$.

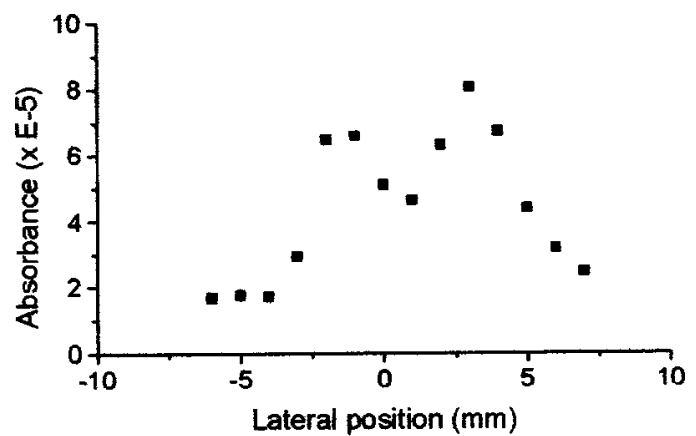

FIG. 8. Ringdown signal intensity vs plasma lateral position. Sr concentration: $25 \mathrm{ppm}$. Microwave power: $120 \mathrm{~W}$.

the plasma $13 \mathrm{~mm}$ above the torch. Absorption maxima appear on either side of the plasma center, indicating that the strongest ringdown absorption is not observed along the diameter of the plasma torch. The signal intensity pattern is slightly asymmetric; this asymmetry is also observed in ICPLIF and ICP-CRDS. ${ }^{28}$ Systematically optimizing physical parameters such as vertical and lateral observation positions and plasma parameters such as gas flow rates, plasma power, etc., can result in significant improvements in detection sensitivity.

\section{Direct comparison with using different light sources (pulsed/CW)}

In order to directly compare the influence of the laser source on the system's detection sensitivity, baseline measurements were also performed with the pulsed laser system operating under the same plasma conditions used to obtain the baseline noise in Sec. IV A. The lowest ringdown baseline noise is $0.26 \%$. Figure 9 presents ringdown time versus $\mathrm{Sr}$ concentration obtained with the pulsed laser. Similarly, the detection limit was determined to be $790 \mathrm{ng} / \mathrm{ml}$, as shown in Fig. 10. These results indicate that using a continuous wave diode laser to replace the pulsed laser system not only reduces the instrument geometry and operating costs, but also quantitatively improves the overall system performance in terms of detection sensitivity.

The comparison of the results obtained using different laser sources shows that the ringdown baseline noise is reduced to $0.2 \%$ from $0.26 \%$ and that the overall detection

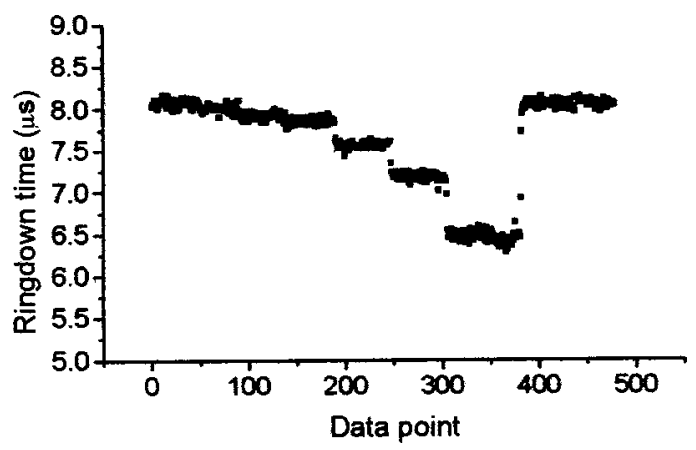

FIG. 9. Ringdown time vs $\mathrm{Sr}$ concentration. The concentration range tested: 0.5-20 ppm. Experimental configuration: pulsed laser with MIP. Light source: pulsed laser system with linewidth $\sim 0.08 \mathrm{~cm}^{-1}$ in the wavelength region used in this work. 


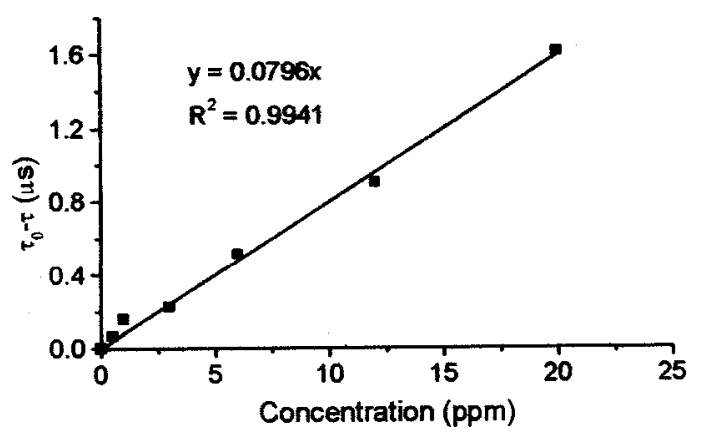

FIG. 10. A typical calibration curve. The detection limit is $790 \mathrm{ppb}$ based on the $3 \sigma$ criteria; $\sigma_{\tau}=0.021 \mu \mathrm{s}$.

sensitivity is improved by a factor of 2 by using the CW laser. It should be noted that the $\mathrm{CW}$ ringdown baseline noise can be readily as low as $0.1 \%^{22,23}$ and even as low as $0.027 \%,{ }^{34}$ which is seven times lower than that measured in this work. Two major reasons are partially responsible for the explanation of the large CW-CRDS baseline noise obtained in the present report. First, the ECDL used in the experiments is still subject to some mode hopping although the manufacturer claims that such a laser is free of the mode hopping. The fine laser frequency modulation is claimed to be achievable through either the current modulation mode or the frequency modulation mode, but it was found that the current modulation mode could not be used for CRDS experiments due to the strong mode hopping observed when implementing this modulation mode (the frequency mode modulation was implemented for the results reported in this work). The mode hopping considerably limits the improvement of the baseline stability. Additionally, this laser is extremely sensitive to the environmental temperature. Two different schemes used to couple the laser beam into the cavity — using a single mode fiber and using a lens system, were implemented for the long time $(>24 \mathrm{~h})$ testing of the laser performance. It was found that the thermal sensitivity of the laser performance affects both the output of the laser power and the spatial orientation of the laser beam. Each of these effects subsequently affects the buildup amplitude of the ringdown signal, so that the trigger level set in the pulse generator has to be frequently readjusted in a typical fourhour experiment. Therefore, the laser performance greatly restricts the improvement of the ringdown baseline noise.

An important consideration in interpreting the results obtained using the both laser sources is the absorption linewidth. The multiple broadening mechanisms at work in the plasma environment typically cause an $\sim 0.4 \mathrm{~cm}^{-1}$ plasmabroadened linewidth (full width at half maximum) for an atom or ion transition. The best sensitivity and accuracy for quantitative ringdown data analysis is obtained when the laser linewidth is much narrower than the absorption feature. ${ }^{54-56}$ With the pulsed laser system used, the ratio of the laser linewidth to the absorption linewidth is $\sim 0.2$. This ratio is fairly good for a weak absorbance if the decay waveform fitting time is set near $3 \tau$, where $\tau$ is the ringdown time on the absorption peak. However, for strong absorption situations, care must be taken in data analysis because multiexponential behavior can result from variation in the absorption cross section across the laser bandwidth. A broader laser linewidth also can reduce sensitivity since some of the laser light is absorbed at less than the maximum cross section of the absorption line. However, with a CW laser source, due to its narrow linewidth, e.g., $<100 \mathrm{MHz}$, such a concern with the linewidth effect on the ringdown quantitative data analysis is negligible.

\section{Estimated detection limits}

As described in Sec. II, the line-of-sight average density of an element in the plasma can be determined from ringdown measurements using Eq. (7) if the absorption crosssection of the elemental transition is known, e.g., determined from Eq. (4). More precisely, the average density of atoms/ ions populating the lower level of the absorption transition can be measured. Correspondingly, the absolute gas phase density of the analyte regardless of the electronic state populated can be estimated by examining how the standard solution is introduced into the plasma and the conversion relation between the liquid phase (e.g., ng/ml) and the gas phase (e.g., atoms $/ \mathrm{cm}^{3}$ ). The conversion relation is described by

$$
\frac{\frac{m c_{s} \eta}{M} N}{V}=n \text {. }
$$

TABLE II. Parameters for converting from liquid phase to gas phase.

\begin{tabular}{|c|c|c|c|}
\hline Definition & Parameter & Used in the work & Unit \\
\hline $\begin{array}{l}\text { Injection rate of } \\
\text { solution sample }\end{array}$ & $m$ & 1.0 & $\mathrm{ml} / \mathrm{min}$ \\
\hline Sample concentration & $c_{s}$ & To be determined & $\mathrm{g} / \mathrm{ml}$ \\
\hline Sample density & $n$ & Measured by CRDS & atom $/ \mathrm{cm}^{3}$ \\
\hline Carrying gas flow rate & $V$ & 0.5 & $\mathrm{~L} / \mathrm{min}$ \\
\hline $\begin{array}{l}\text { Nebulizer conversion } \\
\text { efficiency }\end{array}$ & $\eta$ & 10 & $\%$ \\
\hline Molar mass & $M$ & e.g., U, 238.0289 & g \\
\hline Avogadro constant & $N$ & $6.02 \times 10^{23}$ & $\mathrm{Mol}^{-1}$ \\
\hline Laser beam path-length in the plasma & $l$ & 5 & $\mathrm{~mm}$ \\
\hline $\begin{array}{l}\text { Calculated absorption } \\
\text { cross section }\end{array}$ & $\sigma(\nu)$ & & $\mathrm{cm}^{2}$ \\
\hline $\begin{array}{l}\text { Minimum detectable } \\
\text { absorbance }\end{array}$ & $A=(1-R) 3 \sigma_{\tau} / \tau$ & $9 \times 10^{-6}$ & a.u. \\
\hline
\end{tabular}


Table II lists these parameters along with their definitions used in Eq. (9). Comparing the measured density of absorbers (measured via CRDS) with the estimated total density of the analyte species [estimated using Eq. (9)] allows for the determination of the fraction of the total analyte populating the lower level of the absorption transition.

The estimated total density can also be used to estimate the best possible detection limit of the instrument. As mentioned in Sec. IV A, based on the $3 \sigma$ criteria, the minimum detectable absorbance of the diode laser MIP-CRDS system as currently configured is $\sim 9 \times 10^{-6}$. With knowledge of the absorption cross section of the analyte of interest and the laser beam path-length in the plasma, the minimum detectable density of absorbers can be determined using Eq. (8). Once this minimum density of absorbers is known, the detection limit of the solution sample can then be estimated using Eq. (9). In a previous effort we used this method to successfully estimate the detection limit for lead using ICP-CRDS. ${ }^{26}$ The measured detection limit $(0.3 \mathrm{ng} / \mathrm{ml})$ compared favorably with the estimated best possible detection limit $(0.2 \mathrm{ng} / \mathrm{ml})$, indicating that plasma conditions allowed for most of the lead to be present as ground state atoms. For the current system, this same procedure yields an estimated detection limit of $\sim 0.046 \mathrm{ng} / \mathrm{ml}$ for lead as follows. The difference of a factor of $\sim 4(0.2$ and $0.046 \mathrm{ng} / \mathrm{ml})$ comes from the fact that the baseline noise for the diode laser MIPCRDS system has been reduced to $0.2 \%$ from the $0.5 \%$ found for the pulsed laser ICP-CRDS experiments and the path-length has increased from $3 \mathrm{~mm}$ to approximately 5 $\mathrm{mm}$.

Based on the same procedure, the estimated detection limit of $\mathrm{Sr}$ is $0.001 \mathrm{ng} / \mathrm{ml}$. This number is several orders of magnitude better than the one obtained in the experiment $(375 \mathrm{ng} / \mathrm{ml})$. The major reason that accounts for the large discrepancy is that the measured absorbance is from $\mathrm{SrOH}$ rather than the $\mathrm{Sr}$ element. When a diode laser source is available for the $\mathrm{Sr}$ transition at $461 \mathrm{~nm}$, where $\mathrm{SrOH}$ has no absorption, ringdown measurements of the $\mathrm{Sr}$ element will allow the experimental detection limit to be compared with the theoretical one determined by the procedure described above.

However, in later measurements of uranium using ICPCRDS, this same detection limit estimation yields results that are also orders of magnitude too optimistic. ${ }^{27}$ This is readily understood by considering that the sizable number of lowlying electronic states of either atomic or ionic uranium leads to significant depletion of the lower level of the uranium transition probed even under relatively optimized conditions. In addition, for detection of neutral uranium atoms, the low ionization potential leads to ready loss of atomic population through ionization. The detection limit estimation procedure does not account for thermal excitation and ionization. However, in this regard uranium represents an extreme case, so that the detection limit procedure outlined above remains useful for species with few low-lying transitions.

A better method of estimating detection limits for uranium in the current system is to scale the results obtained in the previous ICP-CRDS studies. ${ }^{27}$ In this way, the complexities of the uranium population distribution are taken into account by previous experiments. Thus, the $107 \mathrm{ng} / \mathrm{ml} \mathrm{detec-}$ tion limit of uranium obtained in ICP-CRDS using the $409.01 \mathrm{~nm}$ transition is expected to improve by a factor of 4 to approximately $25 \mathrm{ng} / \mathrm{ml}$. This estimate does not account for improvements resulting from a narrower laser linewidth or improvements in mirror reflectivity. (The mirrors in the previous work had $R=99.97 \%$ at $409.01 \mathrm{~nm}$.) Of the uranium transitions previously investigated, this transition is highlighted because of its diode laser accessible wavelength. A number of other absorption lines for uranium are available in the blue and red wavelength regions accessible to diodes. Thus, although the size, power, and gas requirements, and cost of the current instrument are far smaller than earlier versions, the performance is expected to improve.

Another improvement in detection sensitivity would result from increasing the effective path-length of the laser beam in the plasma. Exploratory research toward this goal is underway in our laboratories and shows significant promise. A version of compact plasma source is under development to allow the laser beam path-length through the sample region to be more than ten times longer, which should bring about a ten-fold improvement in detection sensitivity. Additionally, the detection sensitivity can be improved by decreasing the baseline noise. In this work, the baseline noise is $0.2 \%$. If a better quality (e.g., mode hop free) diode laser with a higher output power and a lower noise detector are utilized, the baseline noise can be further improved. Even without such improvements, the ability to construct a portable, low power device for elemental and isotopic measurements has been demonstrated.

\section{ACKNOWLEDGMENTS}

The authors are grateful for the financial support of the Office of Science [Biological and Environmental Research (BER)], U.S. Department of Energy under Grant No. DEFG07-02ER63515 at Mississippi State University and Grant No. 86680 at Los Alamos National Laboratory.

${ }^{1}$ A. O'Keefe and D. A. G. Deacon, Rev. Sci. Instrum. 59, 2544 (1988).

${ }^{2}$ J. J. Scherer, J. B. Paul, A. O'Keefe, and R. J. Saykally, Chem. Rev. (Washington, D.C.) 97, 25 (1997).

${ }^{3}$ K. W. Busch and M. A. Busch, editor, ACS Symposium Series 720: Cavity-Ringdown Spectroscopy: An Ultratrace-Absorption Measurement Technique (Oxford University Press, New York, 1999).

${ }^{4}$ G. P. Miller and C. B. Winstead, Cavity Ringdown Laser Absorption Spectroscopy, Invited chapter in Encyclopedia of Analytical Chemistry: Instrumentation and Applications, 10734, edited by R. A. Meyers (Wiley, Chichester, UK, 2000).

${ }^{5}$ G. Berden, R. Peeters, and G. Meijer, Int. Rev. Phys. Chem. 19, 565 (2000).

${ }^{6}$ D. R. Wagner, C. B. Winstead, and G. P. Miller, in Infrared Cavity Ringdown Spectroscopy, in Handbook of Vibrational Spectrsocopy, edited by J. M. Chalmers and P. R. Griffiths, Vol. 1 (Wiley, New York, 2002), pp. 866-880.

${ }^{7}$ D. B. Atkinson, Analyst (Cambridge, U.K.) 128, 117 (2003).

${ }^{8}$ For example, J. J. Scherer, D. Voelkel, D. J. Rakestraw, J. B. Paul, C. P. Collier, R. J. Saykally, and Anthony O'Keefe, Chem. Phys. Lett. 245, 273 (1995).

${ }^{9}$ A. J. Hallock, E. S. F. Berman, and R. N. Zare, Appl. Spectrosc. 57, 571 (2003).

${ }^{10}$ B. A. Paldus, C. C. Harb, T. G. Spence, R. N. Zare, C. Gmachl, F. Capasso, D. L. Sivco, J. N. Baillargeon, A. L. Hutchinson, and A. Y. Cho, Opt. Lett. 25, 666 (2000).

${ }^{11}$ For example, Joshua B. Paul, James J. Scherer, Anthony O'Keefe, L. 
Lapson, J. G. Anderson, C. Gmachl, F. Capasso, and A. Y. Cho, Proc. SPIE 4577, 1 (2001).

${ }^{12}$ A. C. R. Pipino, J. W. Hudgens, and R. E. Hule, Rev. Sci. Instrum. 68 , 2978 (1997).

${ }^{13}$ K. K. Lehmann and P. Rabinowitz, U.S. Patent No. 6,097,555 (2000).

${ }^{14}$ M. Gupta, Hong Jiao, and A. O'Keefe, Opt. Lett. 27, 1878 (2002).

${ }^{15}$ P. B. Tarasa, P. Rabinowitz, and K. K. Lehmann, 224 ACS National Meeting, Boston, MA, 18-22 August 2002 (American Chemical Society, Washington, DC, 2002).

${ }^{16}$ R. S. Brown, L. Kozin, Zhaoguo Tong, R. D. Oleschuk, and Hans-Peter Loock, J. Chem. Phys. 117, 10444 (2002).

${ }^{17}$ D. Romanini, J. Gambogi, and K. K. Lehmann, Proceedings, 50th International Symposium Molecular Spectroscopy, edited by By T. A. Miller (Department of Chemistry, Ohio State University, Columbus, Ohio, 1995), p. 284

${ }^{18}$ D. Romanini, A. A. Kachanov, and F. Stoeckel, Chem. Phys. Lett. 270, 538 (1997)

${ }^{19}$ Y. He and B. J. Orr, Appl. Phys. B: Lasers Opt. 75, 267 (2002).

${ }^{20}$ For example, R. N. Zare, J. Martin, and B. A. Paldus, U.S. Patent No. $5,815,277$ (1998).

${ }^{21}$ R. Augstine, C. R. Krusen, Chuji Wang, and W. B. Yan, U.S. Patent Appl. Publ. Application No. 2002-145209 (2003).

${ }^{22}$ S. T. Scherrer, M.S. thesis, Mississippi State University (unpublished).

${ }^{23}$ Chuji Wang, S. T. Scherrer, and C. B. Winstead (unpublished).

${ }^{24}$ C. B. Winstead, F. J. Mazzotti, J. Mierzwa, and G. P. Miller, Anal. Commun. 36, 277 (1999).

${ }^{25}$ G. P. Miller and C. B. Winstead, J. Anal. At. Spectrom. 12, 907 (1997).

${ }^{26}$ Chuji Wang, F. J. Mazzotti, G. P. Miller, and C. B. Winstead, Appl. Spectrosc. 56, 386 (2002).

${ }^{27}$ Chuji Wang, F. J. Mazzotti, G. P. Miller, and C. B. Winstead, Appl. Spectrosc. 57, 1167 (2003).

${ }^{28}$ Chuji Wang, F. J. Mazzotti, J. Mierzwa, G. P. Miller, Yixiang Duan, and C. B. Winstead, Pittcon 2002, New Orleans, LA (Pittcon, 2002).

${ }^{29}$ Yixiang Duan, Chuji Wang, and C. B. Winstead, Anal. Chem. 75, 2105 (2003).

${ }^{30}$ J. B. Dudek, P. B. Tarsa, A. Velasquez, M. Wladyslawski, P. Rabinowitz, and K. K. Lehmann, Anal. Chem. 75, 4599 (2003).

${ }^{31}$ R. Vasudev, A. Usachev, and W. R. Dunsford, Environ. Sci. Technol. 33, 1936 (1999).
${ }^{32}$ A. R. Awtry and J. H. Miller, Appl. Phys. B: Lasers Opt. 75, 255 (2002).

${ }^{33}$ Chuji Wang and M. J. Plodinec, Breath gas analyzer for diabetes diagnosis and method of thereof, Patent pending No. 10/340,602 (2003).

${ }^{34}$ E. R. Crosson, K. N. Ricci, B. A. Richman, F. C. Chilese, T. G. Owano, R. A. Provencal, M. W. Todd, J. Glasser, A. A. Kachanow, B. A. Paldus, T. G. Spence, and R. N. Zare, Anal. Chem. 74, 2003 (2002).

${ }^{35}$ H. G. C. Human, N. Omenetto, P. Cavalli, and G. Rossi, Spectrochim. Acta, Part B 39, 1345 (1984).

${ }^{36}$ A. Montaser and D. W. Golightly, Inductively Coupled Plasma in Analytical Atomic Spectrometry (VCH Publishers, New York, 1992).

${ }^{37}$ K. K. Lehmann, U. S. Patent No. 5,528,040 (1996).

${ }^{38}$ D. Romanini, A. A. Kachanov, N. Sadeghi, and F. Stoeckel, Chem. Phys. Lett. 264, 316 (1997).

${ }^{39}$ B. A. Paldus, J. S. Harris, Jr., J. Martin, J. Xie, and R. N. Zare, J. Appl. Phys. 82, 3199 (1997).

${ }^{40}$ A. E. Siegman, Laser (University Science Books, Mill Valley, CA, 1986).

${ }^{41}$ G. F. Kirkbright and M. Sargent, Atomic Absorption and Fluorescence Spectrometry (Academic, London, 1974).

${ }^{42}$ NIST, http://physics.nist.gov/cgi-bin/AtData/main_asd

${ }^{43}$ H. R. Griem, Plasma Spectroscopy (McGraw-Hill, New York, 1964).

${ }^{44}$ M. M. Prost, Spectrochim. Acta, Part B 37, 541 (1982).

${ }^{45}$ M. W. Blades, Appl. Spectrosc. 37, 371 (1983).

${ }^{46}$ P. Yang and R. M. Barnes, Spectrochim. Acta, Part B 44, 561 (1988).

${ }^{47}$ S. Nakamura, Spectrochim. Acta, Part B 54, 1899 (1999).

${ }^{48}$ Chuji Wang, F. J. Mazzotti, S. P. Koirala, C. B. Winstead, and G. P. Miller, Appl. Spectrosc. (to be published).

${ }^{49}$ D. S. Baer and R. K. Hanson, J. Quant. Spectrosc. Radiat. Transf. 47, 455 (1992).

${ }^{50}$ R. Peeters, G. Berden, and G. Meijer, Appl. Phys. B: Lasers Opt. 73, 65 (2001).

${ }^{51}$ P. I. Presunka and J. A. Coxon, J. Chem. Phys. 101, 201 (1994).

${ }^{52}$ M. S. Beardah and A. M. Ellis, J. Chem. Phys. 110, 11244 (1999).

${ }^{53}$ R. Mavrodineanu and H. Boiteux, Flame Spectroscopy (Wiley, New York, 1965), p. 380.

${ }^{54}$ P. Zalicki and R. N. Zare, J. Chem. Phys. 102, 2708 (1995).

${ }^{55}$ J. T. Hodges, J. P. Looney, and R. D. van Zee, Appl. Opt. 35, 4112 (1996).

${ }^{56}$ A. P. Yalin and R. N. Zare, Laser Phys. 12, 1065 (2002). 
Review of Scientific Instruments is copyrighted by the American Institute of Physics (AIP). Redistribution of journal material is subject to the AIP online journal license and/or AIP copyright. For more information, see http://ojps.aip.org/rsio/rsicr.jsp Copyright of Review of Scientific Instruments is the property of American Institute of Physics and its content may not be copied or emailed to multiple sites or posted to a listserv without the copyright holder's express written permission. However, users may print, download, or email articles for individual use. 
Review of Scientific Instruments is copyrighted by the American Institute of Physics (AIP). Redistribution of journal material is subject to the AIP online journal license and/or AIP copyright. For more information, see http://ojps.aip.org/rsio/rsicr.jsp 